\title{
Superior Mesenteric Artery Syndrome
}

National Cancer Institute

\section{Source}

National Cancer Institute. Superior Mesenteric Artery Syndrome. NCI Thesaurus. Code C85175.

A very rare syndrome characterized by compression of the third portion of the duodenum against the aorta. The compression is caused by the superior mesenteric artery. It results in complete or partial duodenal obstruction. Signs and symptoms include nausea, vomiting, abdominal pain and distention, failure to gain weight, and weight loss. 